Relations industrielles

Industrial Relations

\title{
Notes en marge d'un projet de recherche sur « L'évaluation méthodique des emplois dans les hôpitaux du Québec »
}

\section{Léo-A. Dorais}

Volume 19, numéro 2, avril 1964

URI : https://id.erudit.org/iderudit/1021313ar

DOI : https://doi.org/10.7202/1021313ar

Aller au sommaire du numéro

Éditeur(s)

Département des relations industrielles de l’Université Laval

ISSN

0034-379X (imprimé)

1703-8138 (numérique)

Découvrir la revue

Citer ce document

Dorais, L.-A. (1964). Notes en marge d'un projet de recherche sur « L'évaluation méthodique des emplois dans les hôpitaux du Québec ». Relations industrielles / Industrial Relations, 19(2), 239-244. https://doi.org/10.7202/1021313ar

Tous droits réservés @ C Département des relations industrielles de l’Université Laval, 1964
Ce document est protégé par la loi sur le droit d'auteur. L'utilisation des services d'Érudit (y compris la reproduction) est assujettie à sa politique d'utilisation que vous pouvez consulter en ligne.

https://apropos.erudit.org/fr/usagers/politique-dutilisation/ 


\section{COMMENTAIRES}

\section{Notes en marge d'un projet de recherche sur «L'évaluation méthodique des emplois dans les hôpitaux du Québec»}

DORAIS, LÉO-A., professeur à l'Université de Montréal.

\section{INTRODUCTION}

Toute recherche empirique mérite d'être considérée avec bienveillance dans un milieu où un tel effort est rare. Ainsi, deux publications récentes du Conseil de Recherche du Comité des Hôpitaux du Québec ${ }^{1}$ méritent un moment d'attention.

Ces deux publications s'insèrent «dans le cadre d'un triptyque » ${ }^{2}$ dont la troisième partie sur «le taux de roulement du personnel hospitalier » est encore en préparation.

Il serait vraiment incorrect d'analyser critiquement ces publications prises comme un ensemble, il faut se contenter d'en examiner une, celle qui porte sur les causes de départ. Il faut de même se garder de faire des rapprochements avec d'autres données qui conduiraient à une critique externe.

Avec ces restrictions en tête, examinons successivement le plan d'ensemble, la méthodologie et l'analyse des résultats de ces travaux. Le lecteur qui aura suivi ces trois étapes saura pourquoi il est inutile d'en commenter les conclusions.

LE PLAN D'ENSEMBLE DE LA RECHERCHE

Partant du principe, fort valable dans sa généralité, "qu'il ne saurait $\mathrm{y}$ avoir de véritable progrès, en administration hospitalière, comme ailleurs, sans de sérieuses recherches $»^{3}$ les auteurs entreprennent des recherches.

(1) Conseil de Recherche de l'Ecole supérieure d'administration hospitalière du Comité des Hôpitaux du Québec, Stabilité du Personnel No 1: Ancienneté du personnel dans les hôpitaux du Québec. (Janvier 1963 selon la page couverture ou octobre 1960 selon la page de garde - le lecteur aura d'ailleurs l'occasion d'exercer ce genre de choix tout au cours de l'ouvrage); Stabilité du Personnel No 2: Les causes de départ des salariés dans les hôpitaux du Québec. (Février $1963)$.

(2) Causes de départ..., p. 5.

(3) op. cit., p. 5. 
Or, il est embarrassant d'entreprendre une recherche sans avoir une théorie générale d'où certaines hypothèses sont tirées et comparées à la réalité. Embarrassant certes, mais non impossible : nos auteurs nous le prouvent en décidant de quantifier.

Cette étude «constitue vraiment de «la» statistique. Elle n'est pas une simple compilation mathématique $\gg^{4}$ Le lecteur s'attend à retrouver les bêtes noires de l'analyse statistique : tests de variance, régressions multiples, analyses factorielles ou autres éléments savants. Il s'imagine mal la surprise que lui préparent les auteurs.

On peut se demander quels sont les buts de l'étude et espérer trouver un résumé clair des objets et des objectifs des auteurs. Le leoteur doit se contenter de lire ${ }^{5}$ ces buts : \& jeter plus de lumière sur certains aspects de l'administration du personnel dans les hôpitaux du Québec» et, «faire une mise au point sur la stabilité du personnel en vue d'y trouver des éléments de solution à d'autres problèmes relevant de l'administration du personnel hospitalier ».

En somme si le texte cité veut dire ce qu'il dit, les auteurs ne s'intéressent pas au problème pour lui-même mais en autant qu'il se rattache à une question plus générale. Mais laquelle? Cela demeure leur secret au grand désespoir du lecteur.

Il s'agit donc d'une étude sur les départs laquelle s'intéresse à ceux-ci en autant qu'ils sont symptomatiques d'autre chose, afin de jeter plus de lumière sur certains aspects de l'administration du personnel hospitalier, lesquels aspects demeurent inconnus.

Si le lecteur de ces lignes est perdu qu'il pense au lecteur du rapport original ...

Il serait possible de suggérer aux auteurs qu'ils ont voulu réaliser une étude descriptive des raisons de départ des salariés dans les hôpitaux du Québec. Ils espèrent que cette étude saura avoir quelque relation avec les problèmes généraux de l'administration du personnel hospitalier. Somme toute, leur recherche n'a pas de base théorique et risque de se relier à aucune idée générale.

Il faut prendre avec bienveillance l'espoir exprimé en ces termes au sujet de cette étude : * elle est susceptible de susciter une revision complète de la politique administrative de nos hôpitaux en matière de bénéfices additionnels au salaire $"{ }^{6}$ Est-ce à dire que les auteurs postulent que le salaire se dissocie des autres bénéfices rattachés à l'emploi? On souhaiterait qu'ils développent davantage cette phrase car

(4) loc. cit., p. 7. Italique dans l'original.

(5) loc. cit., p. 14.

(6) loc. cit., p. 7. 
voici une occasion de se rattacher à un problème plus général : celui de la conception du salaire. Mais, ce genre de demande dépasse le niveau conceptuel de la recherche.

\section{MÉTHODOLOGIE}

Les pages de méthodologie sont riches en noms ${ }^{7}$ mais pauvres de détails quant aux procédures de la recherche.

Le nombre des hôpitaux participant à cette enquête diminue à mesure que le texte progresse. Ainsi, en p. 16 « tous les hôpitaux français du Québec»se réduisent en p. 24 à « cinquante-neuf (59) hôpitaux exactement ont collaboré et nous ont fait parvenir leurs rapports ». En p. 27 on apprend que «six (6) des cinquante-neuf (59) hôpitaux sont à l'extérieur du Québec » et qu'il n'en reste que 53. Mais en p. 29 on doit prendre note «que sur les 53 hôpitaux ...44 nous ont envoyé leurs rapports régulièrement et qu'outre ce nombre 2 nous ont fait parvenir leur rapport annuel complet » Donc en 13 pages le lecteur est passé de tous les hôpitaux français à $\mathbf{4 4}$ qui ont complété tous les documents.

Mais ce nombre doit encore être diminué en considérant le tableau «Départs rapportés par l'hôpital selon le mois et le sexe » en p. 30. On observe d'autres restrictions non-mentionnées à la participation des hôpitaux : 3 n'ont pas fait de rapport un mois, 2 ont négligé deux mois, 2 durant trois mois et 1 durant 4 mois. En considérant ces 8 cas où les résultats sont incomplets, il ne reste que 36 hôpitaux qui ont offert des résultats absolument complets à l'enquête.

Doit-on ici «chicaner» ces enfants terribles qui jouent à « cachecache » avec le lecteur ou leur reprocher de ne rien connaître aux règles de la méthodologie scientifique?

Sentant que ce dernier aspect sera sans doute le réflexe du lecteur averti, ils font chaude gorge du fait que $\ll 30 \%$ de l'ensemble hospitalier du Québec participe ${ }^{8}{ }^{8}$ à l'enquête. (Ce 30\% est par rapport aux 53 hôpitaux non au 36 qui demeurent de fait). Ils soulignent que dans divers domaines * on se contente habituellement d'un échantillon représentant $10 \%$ ou $15 \%$ de la réalité et l'on tire des conclusions $\$ .^{\circ}$ Ils ignorent que cette caractéristique du 10 ou $15 \%$ ne s'applique pas à n'importe quelle population. S'il existe un cas-type de population stratifiée il s'agit bien des hôpitaux : publics ou privés, religieux ou laïques, anglophones ou francophones, spécialisés ou généraux, urbains ou ruraux etc. Or, quel respect a-t-on eu de cette stratification? On a éliminé au départ tous les hôpitaux non-français (c'est un choix qui se justifie sans doute, mais on ne doit pas l'ignorer), on mêle les hôpitaux généraux

(7) op. cit., pp. 15-16.

(8) op. cit., p. 28.

(9) ibidem. 
et spécialisés, les gros et les petits, les hôpitaux urbains et ruraux. Et cette marmalade dont on tire des chiffres représenterait plus que l'échantillon normalement requis? Non, décidément on ignore les canons de la méthodologie scientifique élémentaire.

On peut aussi formuler le regret de voir tant d'énergies gaspillées. Si 10 ou $15 \%$ suffisent, pourquoi avoir $30 \%$ de la population? La raison ici est fort simple, on a oublié de procéder au choix d'un échantillon, acceptant toutes les réponses offertes. Il serait à souhaiter qu'on ait eu davantage conscience des choix impliqués dans l'usage d'une méthode ou d'une autre. Cet aspect est à tel point fondamental qu'il sape toutes les conclusions à leur base. Ces conclusions ne sauraient être valides pour le milieu hospitalier du Québec puisque celui-ci n'est pas représenté comme tel dans l'échantillon.

Même pour les hôpitaux de l'échantillon on peut se demander quelle valeur peut être attachée aux résultats présentés. Ici encore, un aspect méthodologique important: qui fournit les renseignements? On ne sait trop, sinon que ce ne sont certainement pas les employés qui partent. «Dans la majorité des cas, c'est le service du personnel de l'hôpital qui s'est chargé d'enregistrer les données et nous les faire parvenir ». ${ }^{10}$ Dans les autres cas, le lecteur peut imaginer ce qu'il veut comme source des résultats de l'enquête. On ne sait rien de la procédure par laquelle l'employé donne la raison de son départ (on espère que chaque départ a été précédé d'une entrevue), on ne sait rien non plus de la personne qui a eu ce dernier contact avec lui (on s'imagine mal l'employé qui part à cause de difficultés interpersonnelles donner la véritable raison de son départ à l'intéressé), on ne sait pas davantage si les raisons de départ sont offertes à l'employé sous forme de questionnaire à choix multiple ou autrement. L'impression du lecteur devant ces aspects de l'enquête c'est d'avoir plus de questions à poser que de solutions offertes par les auteurs. Il n'est pas question de nier la bonne volonté ni la scrupuleuse honnêteté des personnes qui ont fourni les résultats, il s'agit ici de rappeler aux écoliers que sont ces chercheurs que leur travail narrive pas au niveau promis des «sérieuses recherches».

Bien que le terme «écolier» soit sans doute très fort il semble le plus approprié dans les circonstances. Ces mauvais élèves ont zéro en arithmétique. A ne considérer que les résultats des pages 30 et 33 lesquels résument les départs «par sexe selon le mois 》 on doit signaler que les sommes arithmétiques sont fausses. Le grand total en p. 30 est de 3761 alors qu'il est de 3769 en p. 33. Dans la transposition des données du Tableau I au Tableau II, sur 24 sommes 20 sont incorrectes.

Peut-être que d'autres résultats ont été utilisés dans ces transpositions mais le lecteur n'en sait rien. En tout cas, c'est l'oeuvre d'un mauvais écolier. «Zéro en arithmétique, mon ami!». C'est peut-être cela « la» statistique ...

(10) op. cit., p. 17. 


\section{ANalyse des résultats}

En gardant en tête ce qui précède on s'imagine mal que les auteurs aient le culot d'analyser les résultats obtenus, mais ils le font. Cette analyse consiste à regrouper les données sous diverses têtes de chapitre : dimension d'hôpital, genre d'hôpital, catégorie d'emploi, etc.

Il suffira de s'attacher à un de ces regroupements, « raisons des départs selon les catégories (d'emploi) et en fonction du sexe ${ }^{11}$, en particulier le Tableau XII et le Graphique IV qui conduisent à un corollaire intitulé «L'hôpital est-il responsable ou victime? »

Le corollaire est très révélateur des principes qui ont présidé à toute l'enquête mais qui ne se trouvent pas dans les pages qui contiennent normalement la théorisation des auteurs. Partant du principe que l'hôpital ne saurait être autre chose que victime plutôt que responsable, on s'empresse en deux paragraphes ${ }^{12}$ d'éliminer toute responsabilité de l'hôpital dans $55.5 \%$ des départs. (Notons en passant qu'il y a avantage à ne parler dans le texte qu'en terme de pourcentages - le lecteur en oublie les fausses additions rapportées plus haut). Avec ce pourcentage rassurant, les départs volontaires sont ramenés à $24.8 \%$ des cas dans lesquels l'hôpital a «peu» $(17 \%)$ ou \&une certaine part $(7.8 \%)$ de responsabilité.

Dans les cas de congédiement ( $16.5 \%)$ l'hôpital n'a évidemment $(1 ! !)$ aucune responsabilité alors que dans les mises à pied $(3.08 \%)$ l'hôpital « a un certain rôle».

Ainsi, bon patron, l'hôpital n'aurait qu'à se sentir victime des départs des employés, ce qui d'ailleurs était la conclusion déjà inscrite dans le titre mème de ce corollaire.

« Avant de remédier aux départs du personnel il faut d'abord les connaître d̀ fond et avec exactitude et il faut en savoir les raisons. ${ }^{13}$ Ce travail reste à faire et la fausse sécurité offerte par les pourcentages rapportés plus haut ne peut que nuire au travail d'analyse nécessaire à la négociation collective. Les conditions de travail - salaire et bénéfices additionnels - qui, d'une part, évitent à l'administration les frais impliqués dans les départs et, d'autre part, assurent à l'employé qu'il trouvera satisfaction dans son emploi sont prérequis à cette négociation. Mais comment espérer que cette recherche conduise à une telle ouverture des esprits alors que l'analyse est conçue pour exonorer l'employeur?

C'est ici, il faut le remarquer, que s'inscrit la nécessité de procéder à de telles recherches avec un plan d'ensemble qui se base sur des
(11) op. cit., pp. 84-97.
(12) op. cit., p. 94.
(13) op. cit., p. 99. 
théorisations valables non sur des postulats que l'on retrouve en fin d'analyse. Il est malheureux que ce débat ne puisse s'engager sur un terrain plus adulte, mais avant d'accepter la discussion il faut souligner les insuffisances des prémisses, l'inexactitude des faits et le ridicule de l'effort engagé.

\section{Conclusions}

Si le milieu hospitalier manque de chercheurs et de données objectives valables sur lui-même il faut sans doute en rechercher la cause dans la mauvaise utilisation des ressources financières et de personnel plutôt que leur absence totale. A en croire un autre document relié à l'effort de «recherche» du même groupe ${ }^{14}$ un hôpital estime avoir consacré 52,537 heures de travail à un autre projet. Même à $\$ 1.00$ l'heure cela représente une somme importante qui est difficilement justifiée par l'amateurisme de résultats tels que ceux-ci. On peut imaginer que la « recherche»dont il est question ici a coûté très cher en temps et en argent.

Sans nier l'utilité des groupes de recherche des organismes privés, il semble évident que des ressources égales seraient mieux mises à profit par des organismes universitaires de recherche. Il n'y aurait alors que peu de risques de voir la méthodologie scientifique élémentaire ignorée, l'absence de cadre théorique, le parti-pris patronal teinter l'interprétation, ou encore les données quantitatives rendues invalides par des opérations arithmétiques fautives.

Ce n'est pas dire pour autant que l'auréole universitaire règle automatiquement ces défauts, mais tout au moins permet-elle d'exclure de ses équipes de recherches les pires cancres.

Il n'y a qu'à espérer que les soit-disant résultats de cette « recherche» ne mystifient personne.

(14) L'évaluation méthodique des emplois dans les hôpitaux du Québec: vue d'ensemble et réalisation à date. Octobre 1962. Document miméographié. (Commission de Formation des Chefs de l'Ecole supérieure d'administration hospitalière du Comité des hôpitaux du Québec). p. 10. 